\title{
The Dygogram of Axle Reaction of a Pendulum
}

\author{
By A. G. Greenhill \\ (Read 13th December, 1907. Received, same date.)
}

Dygogram is the name of a curve, invented by Archibald Smith and employed in his Admiralty Manual of the Deviation of the Compass, to give a graphic representation of the varying magnetic field of a compass as the ship is swung round in azimuth; a description is given of the Dygogram by Maxwell in Electricity and Magnetism, $\$ 441$.

There are two kinds of the Dygogram; one gives the magnetic field with respect to the land as the ship turns in azimuth, the other gives the magnetic field relatively to the ship.

The curves are drawn on two cards, superposed and pivoted at a point; and thereby a reading of the compass on the ship can be converted into a true magnetic bearing with respect to the land or chart.

Archibald Smith's Compass Dygogram of the first kind is a Limaçon of Pascal, and the second kind is an ellipse; we may distinguish them as the space and body dygogram.

Now it is curious that the same curve, the limaçon and ellipse, will serve as the dygogram, space and body, for the axle reaction of a pendulum, swinging freely under gravity about a fixed axis.

The investigation is important in the case of a large body, such as a ship, a ballistic pendulum, a church bell, a hammock chair, or a swing at a fair, for calculation of the strength and stability of the frame; and Kater's experimental determination of $g$ by his pendulum is now considered untrustworthy from insufficient consideration of the yielding of the support employed.

The reaction at the axle is obtained at once by replacing the pendulum by two particles kinetically equivalent, in the manner explained in Maxwell's Matter and Motion, CXXI ; one particle of weight $\mathrm{W} \frac{h}{l}$ is placed at centre of oscillation $\mathrm{L}$, and the other of weight $\mathrm{W}\left(1-\frac{h}{l}\right)$ at $\mathrm{O}$ the centre of suspension, $\mathrm{W}$ denoting the 
weight of the pendulum, $l$ the length OL of the equivalent simple pendulum, and $h$ the distance $O G$ from $O$ of $G$ the centre of gravity.

These two particles have the same weight $W$ as the pendulum, the sume centre of gravity at $G$, and the same moment of inertia about $G$

(1) $\mathrm{W} \frac{h}{l}(l-h)^{2}+\mathrm{W}\left(1-\frac{h}{l}\right) h^{2}=\mathrm{W} h(l-h)=\mathrm{W} k^{2}$,

since (2)

$$
l=h+\frac{k^{2}}{h}, l-h=\frac{k^{2}}{h},
$$

$k$ denoting the radius of gyration of the pendulum above an axis through $G$ parallel to the axle through $O$; and so the two particles, if connected rigidly, will in uniplanar motion behave under any force in the same manner as the pendulum.

Of these two particles, the second one acts by dead weight at $O$, where it is at rest; the first particle pulls at the axle with a force in OL, composed in the gravitation unit of $\mathrm{W} \frac{h}{l} \cos \theta$ due to gravity, and $\mathrm{W} \frac{h}{l} \quad \frac{l \omega^{2}}{g}=\mathrm{W} \frac{h \omega^{2}}{g}$ due to centrifugal force of an angular velocity $\omega$; the total pull is then

$$
\mathrm{P}=\mathrm{Wh}\left(\frac{\cos \theta}{l}+\frac{\omega^{2}}{g}\right) \text {. }
$$

Suppose the pendulum to be swinging through an angle $2 a$, so that, by the Principle of Energy,

$$
\begin{gathered}
\frac{1}{2} l \omega^{2}=g(\cos \theta-\cos a) \\
\mathrm{P}=\mathrm{W} \frac{h}{l}(3 \cos \theta-2 \cos a),
\end{gathered}
$$

and the resultant of $P$ in OL and the dead weight $W\left(1-\frac{h}{l}\right)$ vertical at $O$ is the pull of the pendulum on the axle.

Representing $\mathbf{W}$ to scale by the length $l$, then $\mathbf{P}$ is given to the same scale by the vector $r$ of the curve DP in Figure 5, in which

$$
r=h(3 \cos \theta-2 \cos \alpha) \text {, }
$$

a limaçon; and if this curve is drawn with the pole $\mathbf{H}$ vertically below $O$ at a depth $\mathrm{OH}=\mathrm{GL}=l-h$, the pull on the frame of support is given by the vector $O P$; the limaçon DP is thus the 
space, or frame, Dygogram of axle reaction, with respect to a pole at $O$; and as the pendulum swings so that $\theta$ changes from 0 to $\alpha$, the vector $r$ diminishes from $h(3-2 \cos \alpha)$ to $h \cos \alpha$.

Draw PQR vertically upward meeting $O L$ in $Q$, and make $\mathrm{QR}=3 h$; draw $\mathrm{RC}$ perpendicular to $\mathrm{OL}$, then $\mathrm{CQ}=3 h \cos \theta$, while $\mathrm{OQ}=\mathrm{HP}=3 h \cos \theta-2 h \cos a$, so that in Figure 5, where $a$ is an obtuse angle, $\mathrm{OC}=-2 h \cos \alpha$; and $\mathrm{C}$ is the centre of an ellipse APB described by $\mathrm{P}$ in the pendulum, in which $\mathrm{PQ}=\mathrm{OH}=l-h$, and $\mathrm{PR}=l+2 h$ are the semi-axes, minor and major.

The vector PO then represents the pull of the frame on the pendulum, so that the body Dygogram with respect to a pole at $O$ is an ellipse always of the same size, but with the centre $\mathrm{C}$ in OL at a distance $\mathrm{OC}=-2 / \cos a$, depending on $a$ the amplitude of oscillation.

The normal $\mathrm{Pg}^{\prime}$ of the ellipse passes through $g^{\prime}$ the intersection of $\mathrm{Q} g^{\prime}$ parallel to $\mathrm{CB}$ and $\mathbf{R} g^{\prime}$ parallel to $\mathrm{CA}$; or else the tangent VP is given by making $\mathrm{CV}=\mathrm{K} \gamma=(l-h) \operatorname{cosec} \theta$, where $\mathrm{G} \gamma$ is parallel to LK.

The normal $\mathrm{P} g$ of the limaçon is obtained by making $\mathrm{H} g$ equal and parallel to $\mathrm{CR}$.

If a slot $C Q$ and $C R$ is cut on the pendulum, and the point $Q$ and $R$ on a rod $P Q R$ is guided in them as an elliptic compass, so as to be kept vertical by mechanism, the point $P$ will describe the body dygogram ellipse in the pendulum, and the space dygogram limaçon in the frame of support.

At the end of a swing, where

$$
\theta=a, \mathbf{P}=\mathrm{W} \frac{h}{l} \cos a,
$$

so that $P$ is negative if $a>90^{\circ}$, and the thread of a simple pendulum OL would become slack; $\alpha=90^{\circ}$ makes the frame dygogram a circle, and the body dygogram ellipse has a centre at $O$.

When the pendulum just reaches the highest position, $a=180^{\circ}$, $\cos a=-1$; and after the pendulum passes the highest position and makes a complete revolution, - cosa is to be replaced by seca, so that

$$
\mathrm{P}=\mathrm{W} \frac{h}{l}(3 \cos \theta+2 \sec \alpha) ;
$$


and when $2 \sec a=3, a=48^{\circ} 12^{\prime}$, the reaction $P$ is zero at the highest position and $6 \mathrm{~W} \frac{h}{l}$ in the lowest position, and the frame dygogram is a cardioid.

For a larger value of $a$ the frame dygogram is a non-nodal limacon, so that $\mathbf{P}$ does not change sign, as required in a centrifugal railway, where the apparent gravity is nearly zero in the highest position, and so in the lowest position is more than sixfold the ordinary $g$; the carriage does not then leave the track, or the passengers feel any tendency to falling out.

The same conditions are required when wator in a tumbler is whirled round in a vertical circle.

If the vector $O P$ of the body dygogram of a swinging placard or picture falls outside the angle formed at the supporting nail by the two cords, it implies that one of the cords has become slack. So also the vector $O P$ of the frame dygogram of a swing should lie for safety inside the angle formed by the $A$ frame of the supports of the axle.

Measure OI vertically upward of length $\lambda=\frac{g}{\omega^{2}}$, so that $\lambda$ is the height of the equivalent conical pendulum, which swings round the vertical with angular velocity $\omega$; then from (4)

$$
\lambda=\frac{\frac{1}{2} l}{\cos \theta-\cos a},
$$

so that for a swinging pendulum the body locus of $I$ is a hyperbola, with focus at $O$ and direction $l k$ cutting $L O$ produced at right angles in $l$, where $\mathrm{O} l=\frac{1}{2} \mathrm{LO}$; the eccentricity of the hyperbola is sec $a$, and $t \mathrm{I}$ is the tangent at $\mathrm{I}$, if $\mathrm{KO}, k l$ intersect in $t$.

Describe a circle, centre $\mathrm{I}$ and radius IO, cutting $l k$ in $k$; then $I k$ is parallel to an asymptote of the hyperbola, and so makes an angle $a$ with OL.

This hyperbola becomes the straight line $l k$ when $a=90^{\circ}$; and a parabola when $a=180^{\circ}$, and the pendulum just reaches the highest position; afterwards, with complete revolutions, the curve changes into an ellipse.

Draw OK horizontal and LK at right angles to OL to meet in $K$; draw $O J$ at right angles to IK and denote the angle IOJ or OKI by $\phi$. 
Then if $\dot{\omega}$ denotes the angular acceleration of the pendulum, and $\omega$ as before the angular volocity,

$$
\begin{gathered}
\dot{\omega}=\frac{g}{l} \sin \theta=\frac{g}{\mathrm{OK}}, \omega^{2}=\frac{g}{\mathrm{OI}}, \\
\stackrel{\dot{\omega}}{\omega^{2}}=\frac{\mathrm{OI}}{\mathrm{OK}}=\tan \phi ;
\end{gathered}
$$

and the reversed acceleration, or kinetic reaction, at a point $\mathbf{P}$ anywhere on the pendulum is the resultant of $\dot{\omega} \mathrm{OP}$ perpendicular to $\mathrm{OP}$ and $\omega^{2} . \mathrm{OP}$ along $\mathrm{PO}$, and is therefore

$$
\omega^{2} . \mathrm{OP} \sec \phi=g \frac{\mathrm{OP}}{\mathrm{OI} \cos \phi}=g \frac{\mathrm{OP}}{\mathrm{OJ}},
$$

in a direction making an angle $\phi$ with $O P$; and the field of kinetic reaction is thus composed of equiangular spirals of angle $\phi$, with pole common at $O$. Thus the reversal acceleration of $\mathrm{J}$ is $g$, vertically upward.

Combining this field with the uniform field of gravity $g$ vertical, or making an angle $\phi$ with OJ, the resultant field is of strength $g \frac{\mathbf{J P}}{\mathrm{OJ}}$ making an angle $\phi$ with $\mathrm{JP}$, so that the compound field of gravity and kinetic reaction is composed of equiangular spiral lines of force of radial angle $\phi$, round the common pole $J$.

This is the field of force experienced by a body fixed at any point $\mathbf{P}$ of the pendulum; it can be shown experimentally by the varying level of coloured liquid in a small flask fixed at $P$, or by a plummet at the end of a short plumb line thread.

Produce OJ to meet in $\mathbf{J}^{\prime}$ the vertical GNJ' through G, cutting $\mathrm{OK}$ in $\mathrm{N}$; then

$$
\mathrm{OJ}^{\prime} . \mathrm{OJ}=\mathrm{OK} . \mathrm{ON}=\mathrm{OL} . \mathrm{OG}=l h=h^{2}+k^{2}=k_{1}{ }^{2},
$$

where $k_{1}$ denotes the radius of gyration about the axle; thus $J$ and $J^{\prime}$ describe inverse curves with respect to the pole $O$, either in the pendulum or frame.

Drawing $J^{\prime} N^{\prime}$ at right angles to the vertical through $O$, and making $\mathrm{OC}^{\prime}=\mathrm{OC}$,

$$
\begin{gathered}
\mathrm{ON}^{\prime} . \mathrm{OI}=\mathrm{OJ}^{\prime} . \mathrm{OJ}=l h, \\
\mathrm{ON}^{\prime}=\frac{l h(\cos \theta-\cos \alpha)}{\frac{1}{2} l}=2 h(\cos \theta-\cos \alpha),
\end{gathered}
$$

$$
\mathrm{C}^{\prime} \mathbf{N}^{\prime}=2 h \cos \theta, \mathbf{N}^{\prime} \mathbf{J}^{\prime}=h \sin \theta,
$$


so that the frame locus of $J^{\prime}$ is the ellipse, centre $C^{\prime}$ and semi-axes $2 h$ and $h$; and since

$$
\mathrm{GJ}^{\prime}=\mathrm{GN}+\mathrm{ON}^{\prime}=h(3 \cos \theta-2 \cos \alpha)=\mathbf{H P},
$$

the body locus of $\mathrm{J}^{\prime}$ is a limaçon, equal to the frame dygogram DP.

The sensation of the varying compound field is experienced most forcibly on a rolling ship, as shown in Figure 6 by a midship cross section of the Caronia, from keel to flying bridge; the ship is supposed to be rolling through $90^{\circ}$, to $\alpha=45^{\circ}$ on each side of the vertical ; it is drawn when heeled at an angle $\theta=22^{\circ} \frac{1}{2}$.

Considered as a pendulum, the ship may be treated as if supported at $G$, and acted on by the buoyancy, Wtons, applied vertically upward at the metacentre $M$, at a constant metacentric height GM.

The direction of the plumb line is drawn at various positions of $\mathbf{P}$, showing the apparent field of force; if on the flying bridge it will be seen that a body would be left behind in the air if not lashed down, and water would not stay in a tank if open.

Proceeding downward in the ship the motion becomes easier; at $G$ the field of force is undisturbed $g$ vertical; at $L$ the centre of oscillation the direction of the field is along GL, and no motion is felt, or straphanging required, so that water in a vessel at $L$ will not wash about, and the water in a boiler will be almost steady if the surface is not far from $L$.

A swing is of little use as a preparation for a voyage, with the seat in the neighbourhood of $\mathrm{L}$; it must be made like the cross section in Figure 6, with access to the upper parts as high as possible above the axle of suspension.

A model is in use at Woolwich made of canvas stretched on a frame work of battens to resemble figure 2 ; suspended against the wall by a pivot at $M$, the frame can roll like a pendulum, and short plumb lines or small vessels of coloured liquid attached at various points will show the disturbance of the field of apparent gravity.

The canvas is painted to resemble the interior of the ship, like the coloured advertisements; the cross section of the Kaiser Wilhelm $\Pi$. was selected, and made to one-tenth scale; the frame is about 8 feet wide and 12 feet high. 
If our Figure 6 represents a ship of 80 feet beam and $35 \mathrm{draft}$, then GL represents 30 feet, and the time of a single roll from side to side would be about 3 seconds; such a motion would be much too violent for the ship to be habitable, although not unsafe.

The metacentric height GM, about 10 feet as shown in Figure 6, would require to be reduced to about one foot, making GL 300 feet, and the time of a single roll about 9.5 seconds; and now the motion would be comfortable for moderate angle of roll.

A question bearing on the subject proposed by Sir W. Thomson is of historical interest, given in the Mathematical Tripos of 1874, on Thursday afternoon, January 8 , and so it is transcribed here at full length. "In the motion of a compound pendulum prove that

$$
\begin{gathered}
\left(\frac{d \theta}{d t}\right)^{2}=2 \frac{g}{l}(\cos \theta-\cos \alpha), \\
\frac{d^{2} \theta}{d t^{2}}=-\frac{g}{l} \sin \theta
\end{gathered}
$$

showing what $l$ is in terms of properties of the solid and the position of the axis.

Find the force which acts on any infinitesimal part of the whole mass, to balance its weight and give it its acceleration. Hence show that if an infinitely small mass be hung by an infinitely short cord from any point $(x, y)$ of the pendulum, the indication of the cord to $x$ will be

$$
\tan ^{-1} \frac{-g \sin \theta-\dot{\omega} x+\omega^{2} y}{g \cos \theta+\dot{\omega} y+\omega^{2} x},
$$

the axis of $x$ being the line through the point of support and the centre of gravity of the pendulum; and $\omega, \dot{\omega}$ being used to denote $\frac{d \theta}{d t}$ and $\frac{d^{2} \theta}{d t^{2}}$ respectively.

Supposing a ship to roll round a horizontal axis according to the same law as that of the vibration of a compound pendulum, find the pasition of the axis and the angle (great or small) through which the ship rolls; from observations on two plummets hung by very short threads at a given distance from one another 
in a line through the axis perpendicular to the deck, and a determination of the period of rolling in cases where the angle is small."

The Dygogram can be illustrated experimentally by balancing an object on the floor or table, like a poker, a ruler, a circular protractor, or a pin; letting it fall over, and observing whether the contact is broken.

Changing the measurement of $\alpha$ and $\theta$ to deviation from the upward vertical, the height of the shoulder of the frame Dygogram limaçon above $\mathrm{H}$ is $\frac{1}{3} h \cos ^{2} \alpha$, and then $r=h \cos \alpha, \cos \theta=\frac{1}{3} \cos \alpha$; and $O$ lies below the shoulder where

$$
\begin{aligned}
& l-h=\frac{k^{2}}{h}=r \cos \theta=h\left(2 \cos \alpha \cos \theta-3 \cos ^{2} \theta\right) \\
& 3 \cos \theta=\cos \alpha \pm \sqrt{ }\left(\cos ^{2} \alpha-3 \frac{k^{2}}{h^{2}}\right) \\
& r=h \cos \alpha \mp \sqrt{ }\left(h^{2} \cos ^{2} \alpha-3 k^{2}\right) .
\end{aligned}
$$

This does not occur unless

$$
h^{2} \cos ^{2} \alpha>3 k^{2},
$$

and between these values of $r$ and $\theta$ the vector $O P$ of the Dygogram points upward, and contact is broken unless the axle is held down by the cap of a bearing, as in a church bell.

For a uniform rod, like a flat ruler, $k^{2}=\frac{1}{3} h^{2}$, so that $O$ is at the level of the shoulder of the limaçon when $a=0$, and the ruler is balanced on one end in the vertical position, and then the contact is broken for an instant when $\cos \theta=\frac{1}{3}, \theta=70^{\circ} \frac{1}{2}$.

A circular disc, like a protractor, $k^{2}=\frac{1}{4} a^{2}$, just breaks contact if let fall from an angle $a=30^{\circ}$; if balanced vertically, contact is broken when $\theta$ reaches $60^{\circ}$.

A pin will show a difference according as it is balanced on the point or the head, and then let fall. The demolition of a tall chimney stalk will provide an illustration on a large scale, as to whether contact with the ground is broken, or not.

With $h=l$, the pendulum becomes the simple pendulum of a thread OL and plummet $L$, and $O$ then coincides with $H$, the node of the Dygogram limaçon. 
The thread becomes slack when $\cos \theta=\frac{2}{3} \cos \alpha$; but this does not happen unless $a>90^{\circ}$, or for complete revolutions when $\cos \theta=-\frac{2}{3} \sec \alpha$.

The plummet then proceeds to describe a parabola, osculating the circle; and the thread becomes tight again where the circle and parabola intersect, which will be on the isoclinal chord; so that if $\theta$ is the angle the thread makes with the upward vertical when the thread becomes slack, $3 \theta$ is the angle when the thread tightens again.

The extension of the Dygogram is not difficult to the case of a symmetrical top, spinning with the point in a smooth cup, or suspended from a universal joint ; an additional component force is introduced of magnitude

$$
\mathrm{W} \frac{\mathrm{C}}{\mathrm{A}} \frac{h \mathrm{R} \omega}{g},
$$

along the vector of $\omega$ the component angular velocity perpendicular to the axis of figure of the top, where C,A denote the moment of inertia about the axis of the top and about a perpendicular axis through the point, and $R$ denotes the angular velocity of the spin.

A similar formula holds if the pendulum contains a flywheel, and it will serve to explain the theory of the Schlick apparatus for mitigating the rolling of a ship. 